$$
\begin{aligned}
&\left\{\frac{d P}{d t}\right\}^{T}[B]-\left\{\frac{\partial T}{\partial q}\right\}^{T}[B]=\{Q\}^{T}[B] \\
&+\{\sigma\}^{T}\left[\left[A_{1}\right]\left[A_{2}\right]\right][B],
\end{aligned}
$$

由于

$$
\begin{aligned}
\{\sigma\}^{T}\left[\left[A_{1}\right]\left[A_{2}\right]\right][B] & =\{\sigma\}^{T}[[I][O]] \\
& -\left[\{\sigma\}^{T}\{O\}^{T}\right],
\end{aligned}
$$

于是得到

$$
\begin{aligned}
\left\{\frac{d P}{d t}\right\}^{T}[B] & -\left\{\frac{\partial T}{\partial q}\right\}^{T}[B]-\{Q\}^{T}[B] \\
& +\left[\{\sigma\}^{T}\{O\}^{T}\right],
\end{aligned}
$$

式中 $\{0\}$ 为 $(n-s) \times 1$ 零矩阵, $\{\sigma\}^{T}=$ $\left[\sigma_{1} \cdots \sigma_{1}\right]$, 这就是非线性非完整系统的标准 化的 Routh 方程,它包含了消去待定乘子的
运动方程以及关于待乘子已解佣的方程. 由 于所给出的运动方程已消去了未知的待定乘 子,因此能方便地用来求解运动; 又由于络出 的和约束反力有关的方程关于待定乘子是已 解耦的, 因此可以直接用来求解各个约束反 力. 由此可知, 本文所给出的标准化的 Routh 方程, 可以用来求解复杂的系统工程的运 动规律与约束反力.

\section{美文 虾}

[1] 孙右烈, 非线性非完鳌系统的运动方程及其广义偣 量积分,上海力学, $9(1988), 3: 23-28$.

孙右烈

(上海工业大学力学系)

\title{
有限集合上函数的强等价类
}

假设 $D 、 R$ 是有限集,记 $R^{D}-\{f ; f: D \rightarrow$ $R$ \}. 又假设 $G 、 H$ 是分别作用在 $D 、 R$ 上的 置换群. L. Carlitz 定义 $R^{D}$ 中的强等价关 系如下：对于任意的 $f 、 g \in R^{D}$ ，称 $f$ 关于 $G 、 H$ 强等价于 $g$ ，如果存在 $\sigma \in G$ 和 $\tau \in H$ 使 得 $f(\sigma d)-g(d)$ 和 $\tau f(d)-g(d)$ 对任一 $d \in D$ 成立 (Amer. Math. Soc., 75(1953), 405-427). S. R. Cavior 讨论了当 $G=$ $H-S_{n}$ （n 次对称群）的特殊情形（Acta Arith., 1964，10，119-136). 我们得出了 一般情形的公式, 并给出了具有最大数目的 强等价类的特征刻划。

对于 $f_{i} \in R^{D}$, 记 $F_{i}-\left\{f \in R^{D} ; f-f_{i} \sigma\right.$, $\sigma \in G\}, H\left(f_{i}\right)-\left\{\tau \in H ; \tau f_{i}=f_{i} \sigma, \sigma \in G\right\}$ 和 $H_{0}\left(f_{i}\right)-\left\{\tau^{\prime} \in H ; \tau^{\prime} f_{i}-f_{i}\right\}$.

定理 1 用 $N(D, R, G, H)$ 记 $R^{D}$ 中 关于 $G 、 H$ 的强等价类的个数, 则

$$
N(D, R, G, H)-\sum_{t \in R^{D}} \frac{1}{\left[H(f): H_{0}(f)\right]} .
$$

\section{文阶 Heisenberg 方程的 Lax 表示}

L. A. Taktajan (Phys. Lett., $64 \mathrm{~A}$ (1977)，235) 给出了连续的 Heisenberg 旋
定理 2 假设 $D-\{0,1,2, \cdots, n-$ 1\}, $R=\{0,1,2, \cdots, m-1\}, S_{k}$ 为 $k$ 次对 称群, 则

$$
\begin{aligned}
& N\left(D, R, S_{n}, S_{m}\right) \\
& -\sum(m ! n !) /\left\{\prod_{i=1}^{k}\left(n_{i j} !\right)^{m_{i j}}\right. \\
& \left.\quad \cdot \prod_{i=1}^{k}\left(m_{i j} !\right)^{2} \cdot\left[m-\sum_{i=1}^{k} m_{i j}\right] !\right\} .
\end{aligned}
$$

定理 3 假设 $D-\{0,1,2, \cdots, n-$ $1\}, R=\{0,1,2, \cdots, m-1\}, R^{D}-\{f ; f: D \rightarrow$ $R\}$. 又假设 $G$ 是包含循环群 $C$. 在内的置换 群, $G$ 作用在 $D$ 上. $C_{m}$ 是循环群作用在 $R$ 上, 则

$$
N\left(D, R, G, C_{n}\right)-m^{n} \text { 的充分必要条件 }
$$
是: $m 、 n$ 的最大公因子 $(m, n)-1$.

C. Y. Chao

(University of Pittoburgb, USA)

靽绍岑

（四川帅范学睆数学系,南充）

转链方程的 Lax 表示, D. Y. Chen 和 Y. S. Li (Acta Math. Sinica, 2 (1986), 343) 
得到了高阶 Heisenberg 方程. 本文首先给 出高阶 Heisenberg 向量场的换位子表示,进 而导出高阶 Heisenberg 方程的 Lax 表示。

考虑谱问题

$\psi_{x}-U \phi, U=\left(\begin{array}{rr}w & u \\ v & -w\end{array}\right)(-i \zeta)$,

$w^{2}+u v-1$.

命医 1 (1) 式等价于 $L \psi-\zeta \psi$, 共 中 $L=i W \partial, W=\left(\begin{array}{rr}w & u \\ v & -w\end{array}\right), \partial-\frac{\partial}{\partial x}$.

命勇 2

$$
\begin{aligned}
L_{t} & =\frac{d}{d t} L-i\left(\begin{array}{cc}
-\frac{v u_{t}+u v_{t}}{2 \omega} & u_{t} \\
v_{t} & \frac{v u_{t}+u v_{t}}{2 \omega}
\end{array}\right) \partial, \\
L_{*} & \left(\begin{array}{c}
\delta u \\
\delta v
\end{array}\right)-\left.\frac{d}{d \theta}\right|_{s w 0} L(u+8 \delta u, v+8 \delta v) \\
& -i\left(\begin{array}{cc}
-\frac{v \delta u+u \delta v}{2 \omega} & \delta u \\
\delta v & \frac{v \delta u+u \delta v}{2 \omega}
\end{array}\right) \partial .
\end{aligned}
$$

设 $G_{1}(x), G_{2}(x)$ 为任意光滑函数, $G=$ $\left(G_{1}, G_{2}\right)^{T}, \nabla=\left(\begin{array}{ll}A_{1} & B_{1} \\ C_{1} & D_{1}\end{array}\right) \partial$, 按曹策问关于 向量场的换位表示的框架，我们希望得到

$$
[\hat{\nabla}, L]-L_{*}(K G)-L_{*}(J G) L,(2)
$$

其中 $\boldsymbol{K}, J$ 为待定的 Lenard 算子对. (2)式 可变形为

$$
[V, L]=L_{*}(K G) L^{-1}-L_{*}(J G),(3)
$$

其中 $L^{-1}=-i \partial^{-1} W, \partial \partial^{-1}=\partial^{-1} \partial-1$, $V-\hat{V} L^{-1} \triangleq\left(\begin{array}{ll}A & B \\ C & D\end{array}\right)$.

命题 3 设 $B(x) 、 C(x)$ 为任意光滑 函数, $G(x)-(B(x), C(x))^{x}$, $V-V(u, v, B, C)-$

$\left(\begin{array}{cc}-\frac{1}{2} \partial^{-1}\left(\frac{u}{w} C^{\prime}+\frac{v}{w} B^{\prime}\right) & B \\ C & \frac{1}{2} \partial^{-1}\left(\frac{u}{w} C^{\prime}+\frac{v}{w} B^{\prime}\right)\end{array}\right)$,

则等式(3)成立,其中

$$
J=\left(\begin{array}{cc}
-u \partial^{-1} \frac{v}{w} \partial-2 w & -u \partial^{-1} \frac{u}{w} \partial \\
v \partial^{-1} \frac{\nu}{w} \partial & v \partial^{-1} \frac{u}{w} \partial+2 \omega
\end{array}\right) \text {, }
$$

$$
K=i\left(\begin{array}{ll}
\partial & 0 \\
0 & \partial
\end{array}\right) .
$$

递归定义 Lenard 序列: $G_{-1}-(u, v)^{T}$, $K G_{i-1}-{ }_{J} G_{i}, i-0,1,2, \cdots, X_{i}={ }_{J} G_{i}$ 称. 为 Heisenberg 向量场.

定理 1 设 $G_{i}$ 为 Lenard 序列, $V_{i}=$ $V\left(u, v, B_{i}, C_{i}\right)$, 则 Heisenberg 向量场有换 位子表示:

$$
\left[W_{m}, L\right]-L_{*}\left(X_{m}\right),
$$

其中 $W_{m}-\sum_{i=-1}^{m-1} \hat{\nu}_{i} L^{m-1-i}, \hat{v}_{i}-V_{i} L$.

定理 $2\left(\begin{array}{l}u \\ v\end{array}\right),-X_{t}$ 当且仅当 $L_{s}-$ $\left[W_{n}, L\right]$.

定理 3 高阶 Heisenberg 方程是下 述 Lax 对的相容性条件: $L \psi-\zeta \psi, \phi_{t}-$ W. $\psi$.

注 1. 前几个计算结果:

$$
G_{-1}-\left(\begin{array}{l}
u \\
v
\end{array}\right),
$$

$G_{0}=$

$\frac{1}{2 i}\left(\begin{array}{l}\frac{1}{w} u^{\prime}-\frac{1}{2} u \partial^{-1} v \partial \frac{u^{\prime}}{w}+\frac{1}{2} u \partial^{-1} u \partial \frac{v^{\prime}}{w} \\ -\frac{1}{2} v \partial^{-1} v \partial \frac{u^{\prime}}{w}-\frac{1}{w} v^{\prime}+\frac{1}{2} v \partial^{-1} u \partial \frac{u^{\prime}}{w}\end{array}\right)$,

$X_{0}=\left(\begin{array}{l}u^{\prime} \\ v^{\prime}\end{array}\right), X_{1}-\frac{1}{2}\left(\begin{array}{l}u^{\prime \prime} w-u w^{\prime \prime} \\ v w^{\prime \prime}-w v^{\prime \prime}\end{array}\right)$,

其中 $\left(\begin{array}{l}u \\ v\end{array}\right)_{s}-X_{1}$ 为 Heisenberg 旋转链方程.

注 2 .

$$
\begin{aligned}
\mathscr{L} & =J^{-1} K \\
& =\frac{1}{2 i}\left(\begin{array}{l}
\frac{1}{w} \partial-\frac{1}{2} u \partial^{-1} \nu \partial \frac{1}{w} \theta \\
-\frac{1}{2} \nu \partial^{-1} \nu \partial \frac{1}{w} \partial \\
\frac{1}{2} u \partial^{-1} u \partial \frac{1}{w} \partial \\
\\
-\frac{1}{w} \partial+\frac{1}{2} v \partial^{-1} u \partial \frac{1}{w} \partial
\end{array}\right)
\end{aligned}
$$

称为 Lenart 递推算子.

许太喜 顾祝全 (石家庄铁道学院) 